\title{
ING3 and ING4 immunoexpression and their relation to the development of benign odontogenic lesions
}

Yailit del Carmen Martinez-Vargas (1, Tiago João da Silva-Filho ${ }^{2}$, Denise Hélen Imaculada Pereira de Oliveira ${ }^{3}$, Rani Iani Costa Gonçalo Q1', Lélia Maria Guedes Queiroz@1.

The Inhibitor of Growth (ING) gene family is a group of tumor suppressor genes that play important roles in cell cycle control, senescence, DNA repair, cell proliferation, and apoptosis. However, inactivation and downregulation of these proteins have been related in some neoplasms. The present study aimed to evaluate the immunohistochemical profiles of ING3 and ING4 proteins in a series of benign epithelial odontogenic lesions. Methods: The sample comprised of 20 odontogenic keratocysts (OKC), 20 ameloblastomas (AM), and 15 adenomatoid odontogenic tumors (AOT) specimens. Nuclear and cytoplasmic immunolabeling of ING3 and ING4 were semi-quantitatively evaluated in epithelial cells of the odontogenic lesions, according to the percentage of immunolabelled cells in each case. Descriptive and statistics analysis were computed, and the $p$-value was set at 0.05. Results: No statistically significant differences were found in cytoplasmic and nuclear ING3 immunolabeling among the studied lesions. In contrast, AOTs presented higher cytoplasmic and nuclear ING4 labeling compared to AMs (cytoplasmic $p$-value $=0.01$; nuclear $p$-value $<0.001$ ) and OKCs (nuclear $p$-value $=0.007$ ). Conclusion: ING3 and ING4 protein downregulation may play an important role in the initiation and progression of more aggressive odontogenic lesions, such as AMs and OKCs.

\section{Introduction}

A large variety of lesions arises in the maxillary bones. Among them, cysts and odontogenic tumors, which originate from epithelial cell remnants of the dental germ (1). Regarding benign odontogenic lesions, odontogenic keratocysts (OKCs) and ameloblastomas (AMs) are frequently associated with their aggressive behaviors and high recurrence potential (2). For this reason, despite the fact that $\mathrm{OKC}$ presents a cystic nature, many studies are currently underway to clarify the molecular mechanisms involved in the peculiar behavior of this lesion (3). On the other hand, adenomatoid odontogenic tumors (AOTs) are characterized by its indolent clinical and biological behavior (4).

Despite its etiology, the biological behavior of any lesion is not only highly dependent on its proliferative activity, but also on cell death rates. Proteins from the tumor suppressor gene family, called Inhibitors of Growth (ING), have been associated with cell cycle control, cell senescence, replication, DNA repair, cell proliferation, apoptosis and angiogenesis. In several malignancies, ING proteins appear inactivated or downregulated, hence, being classified as class II tumor suppressor genes (5). In humans, five members of the ING family have been identified: ING1, ING2, ING3, ING4 and ING5 $(6,7)$. In contrast to ING1 and ING2, which functionality is already well stablished, conflicting data have been reported concerning ING3 and ING4 role in neoplasms (8).

Inactivation and reduction of ING gene expression generally occurs at the RNA level and have been reported in human lung, breast, gastric, esophagus and brain cancers (5). Additionally, reduced expression of ING proteins in head and neck squamous cells carcinomas has also been reported, which may indicate a more aggressive pattern (9). In melanomas, nuclear ING3 expression is reduced and may be an independent prognostic factor (10). Similarly, low levels of ING4 expression have been correlated with high-grade tumors and poorer prognoses in certain malignant neoplasms, including melanoma, gastric adenocarcinoma, and hepatocellular carcinoma (6). Thus, ING4 may play a role as a tumor suppressor in different tissues, since it appears to be unfunctional in many pro-neoplastic mechanisms (11). 
In the context of cancer, ING levels have been well characterized (11). However, little attention has been paid to ING family expression in benign tumors. Considering the involvement of tumor suppressor genes in the development of tumors, in order to better understand the role of these markers in the development and biological behavior of odontogenic lesions, the present study aimed to evaluate the immunohistochemical profiles of ING3 and ING4 proteins in a series of benign epithelial odontogenic lesions.

\section{Materials and methods}

\section{Ethical considerations}

The Research Ethics Committee of the institution (Approval No. 1,277,582) approved the study.

\section{Sample}

Among all cases of benign odontogenic lesions diagnosed at a Laboratory of Oral Pathology, we selected 55 specimens, which included 20 OKC, 20 AM and 15 AOT. In order to prevent the loss of material antigenicity, all cases that have undergone decalcification processes were excluded from the study. Further, two oral pathologists reviewed the histopathological diagnosis of each case.

\section{Immunohistochemistry}

For the immunohistochemical study, $3-\mu \mathrm{m}$-thick sections were obtained from paraffin-embedded tissue blocks. The tissue sections were deparaffinized and immersed in $3 \%$ hydrogen peroxide to block endogenous peroxidase activity. The slides were then washed in phosphate-buffered saline (PBS). ING3 antibody was polyclonal (clone 30330002; Novus Biologicals, Littleton, CO, USA), used at a dilution of 1:4000. Antigenic recovery was in $10 \mathrm{mM}$ sodium citrate, $\mathrm{pH}$ 6.0, during 30 minutes, followed by overnight incubation at $4{ }^{\circ} \mathrm{C}$. Concerning ING4, a polyclonal antibody (clone NBP2-33828; Novus Biologicals) also used in a dilution of 1:4000, with the same specifications of antigenic recovery and incubation time as the previously mentioned antibody.

After treatment with normal serum, tissue sections were incubated in a moist chamber with primary antibodies. Then, the tissue was washed twice in PBS and treated with a polymer-based complex, at room temperature, to bind the primary antibodies. Peroxidase activity was visualized by immersing tissue sections in diaminobenzidine (Liquid DAB + Substrate; Dako, Carpinteria, CA, USA), resulting in a brown reaction product. The tissue sections were then counterstained with Mayer's hematoxylin and cover slipped.

As positive controls for the reactions, oral squamous cell carcinoma and dental follicle, specimens were used for the ING3 and ING4 (Figure 1). The negative control consisted of Bovine serum albumin in PBS without antibody.

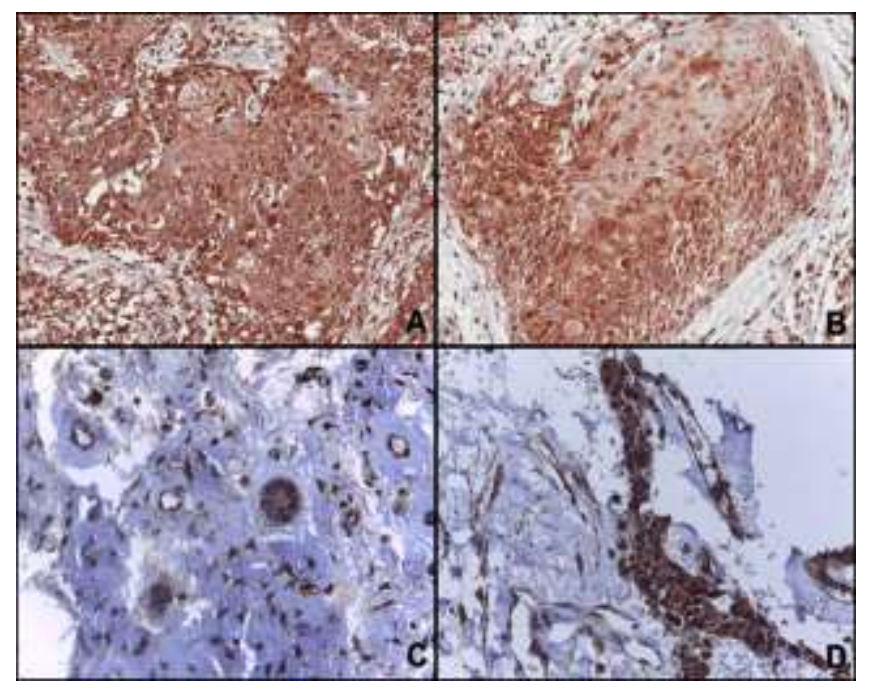

Figure 1. Positive controls for ING3 and ING4 immunolabeling in specimens of oral squamous cell carcinoma (OSCC) and dental follicle. (A) ING3 expression in OSCC. (B) ING4 expression in OSCC. (C) ING3 expression in dental follicle. (D) ING4 expression in dental follicle (IHC; 100x magnification). 


\section{Immunolabeling assessment and statistical analysis}

Immunolabelled slides were scanned using a Panoramic MIDI Scanner (3DHISTECH Kft 2933, Konkoly-Thege M. str. Budapest, Hungary), and the images obtained were observed through the Panoramic Viewer software 1.15.2 (3DHISTECH Kft 29-33, Konkoly-Thege M. str. Budapest, Hungary). Immunoblots of each case were analyzed semi-quantitatively in a double-blind study, by two previously trained evaluators, and aspects related to the antibodies used in odontogenic epithelial cells were verified.

Under a $100-\mu \mathrm{m}$ scale bar, five representative and random fields were determined for each of the evaluated antibodies. Nuclear and cytoplasmic immunoexpression for ING3 and ING4 were then evaluated in epithelial cells of the odontogenic lesions. Subsequently, each case was categorized according to labeling levels, according to the following cell scores, as recommended by Wang et al. (9) and Vered et al. (12): 0 - absent (no positive cells); 1- low (1 to 10\% of positive cells); 2 - intermediate (11-50\% of positive cells); and $3-$ high (> 50\% of positive cells).

\section{Statistical analysis}

The results were analyzed using OriginPro 2016 (OriginLab Corporation ${ }^{\circledR}$ ). Descriptive analysis is presented in absolute and relative frequencies. In order to compare protein expression between lesions, Kruskal-Wallis and Mann Whitney tests were performed. All tests were performed at a 5\% significance level $(p>0.05)$.

In addition, Cohen's Kappa coefficient was calculated to measure the inter-observer agreement, which denoted a good agreement $(\kappa=0,772)$.

\section{Results}

\section{ING3 immunohistochemical expression}

ING3 protein was diffusely expressed in the cytoplasm of epithelial cells of all analyzed cases, regardless of lesion type (Figure 2). Based on the analysis of the positive control specimens, AM and OKC groups exhibited more cases with higher cytoplasmic immunolabeling when compared to the AOT group. In regard to nuclear immunolabeling, in the majority of AM and OKC cases, epithelial cells exhibited reduced expression when compared to controls. However, no statistically significant differences were detected in ING3 cytoplasmic $(p$-value $=0.888)$ and nuclear $(p$-value $=0.06)$ evaluation between the analyzed groups (Table 1).

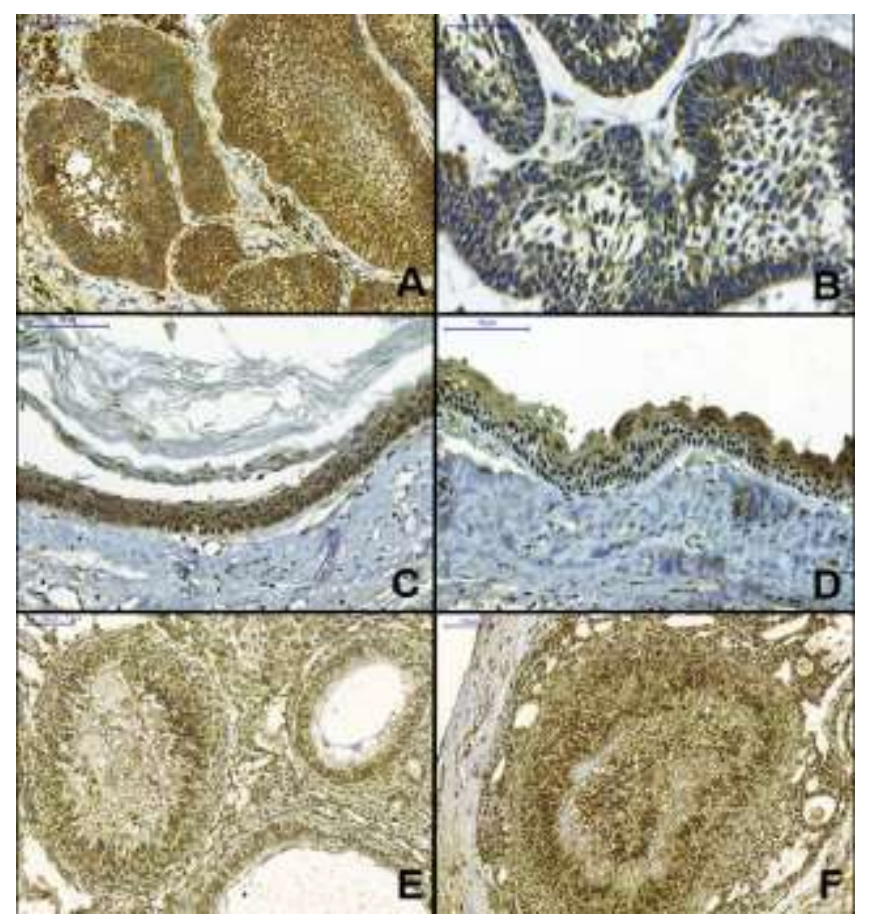

Figure 2. ING3 cytoplasmic and nuclear immunolabeling in AM, OKC and AOT. (A) High cytoplasmic and low nuclear labeling on AM tumor cell islands. (B) Loss of expression in the epithelial component of AM. (C) High cytoplasmic immunolabeling in the epithelial lining of OKC. (D) Absence of both nuclear and cytoplasmic immunolabeling in the epithelial lining of OKC. (E)/(F) Moderate-high cytoplasmic and nuclear staining in AOT tumor parenchyma cells (IHC). 
Table 1. Sample size, median, quartiles 25 and 75, mean of the ranks, Kruskal-Wallis statistic and statistical significance (p) for ING3 immunolabeling scores (cytoplasmic and nuclear labeling) in relation to the groups.

\begin{tabular}{|c|c|c|c|c|c|c|}
\hline Location/Groups & $\mathbf{N}$ & Median & $\mathbf{Q}_{25}-\mathbf{Q}_{75}$ & Mean ranks & $\mathbf{H}$ & $\mathbf{P}$ \\
\hline \multicolumn{7}{|l|}{ Cytoplasm } \\
\hline $\mathrm{AM}$ & 20 & 3.00 & $2.00-3.00$ & 29.15 & 0.2363 & 0.888 \\
\hline OKC & 20 & 3.00 & $2.00-3.00$ & 31.175 & & \\
\hline AOT & 15 & 3.00 & $2.00-3.00$ & 31.175 & & \\
\hline \multicolumn{7}{|l|}{ Nucleus } \\
\hline $\mathrm{AM}$ & 20 & 1.00 & $0.00-1.75$ & 23.025 & 5.36 & 0.06 \\
\hline $\mathrm{OKC}$ & 20 & 1.00 & $0.25-2.00$ & 27.625 & & \\
\hline AOT & 15 & 2.00 & $1.00-3.00$ & 35.13 & & \\
\hline
\end{tabular}

Legend: AM - ameloblastoma; OKC - odontogenic keratocyst; AOT - adenomatoid odontogenic tumor; $\mathrm{H}$ - Kruskal-Wallis coefficient.

\section{ING4 immunohistochemical expression}

Analysis of cytoplasmic ING4 immunolabelling in epithelial cells revealed positivity in all OKC, AM, and AOT cases (Figure 3). Predominance of intermediate (score 2) and high (score 3) expression were observed in the AM and OKC groups, respectively. In contrast, in the AOT group, the scores 2 and 3 were equally distributed.

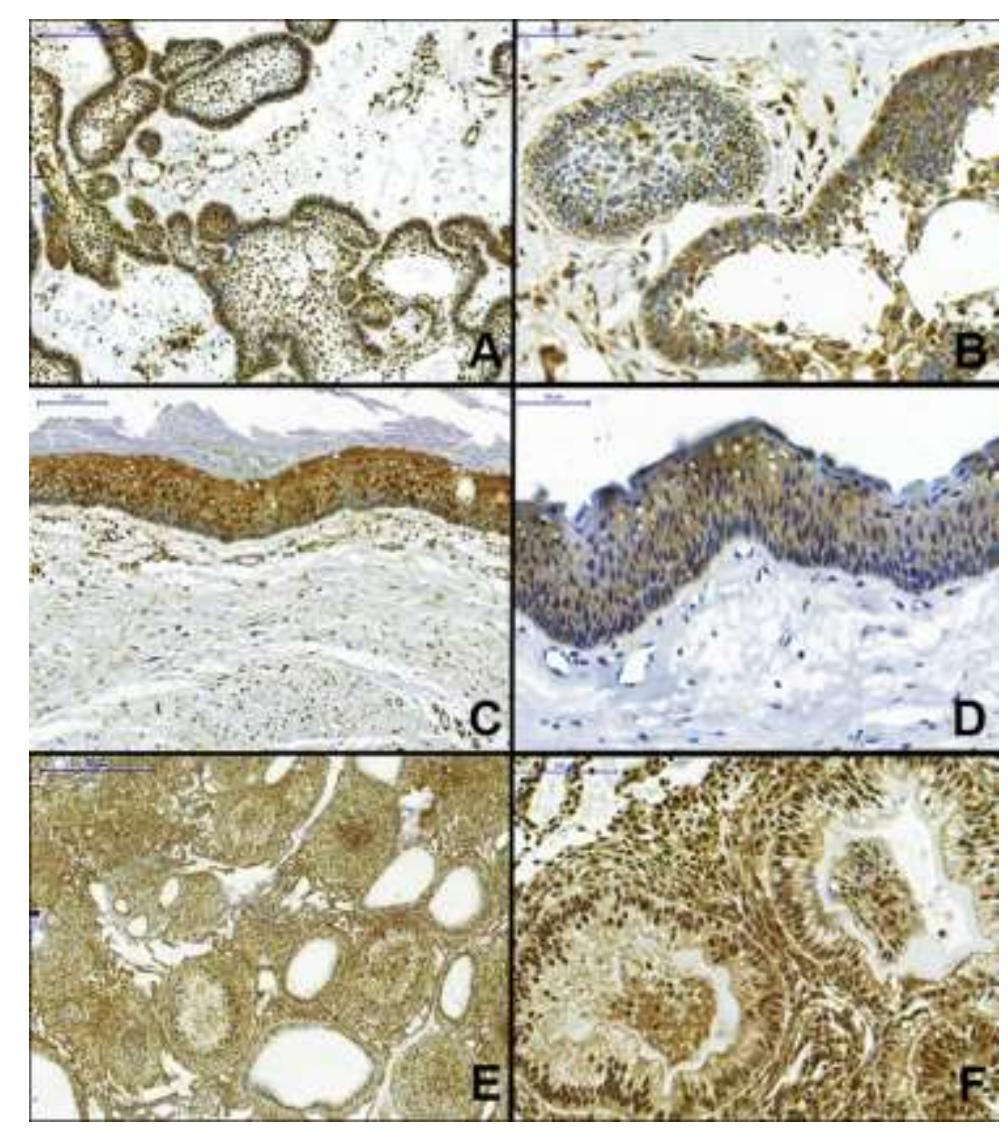

Figure 3. ING4 cytoplasmic and nuclear immunolabeling in AM, OKC and AOT. (A) Moderate cytoplasmic immunolabelling in AM. (B) Low nuclear expression in the epithelial component of AM. (C) OKC showing high cytoplasmic expression of ING4. (D) Absence of nuclear immunolabeling in the epithelial lining of OKC. $(\mathrm{E}) /(\mathrm{F})$ High cytoplasmic and nuclear immunolabeling in AOT tumor parenchyma cells $(I H C)$ 
Based on positive controls, the analysis of cytoplasmic labeling of ING4 revealed that AOTs presented higher labeling compared to AMs $(p$-value $=0.001)$ and OKCs ( $p$-value $=0.490)$. AMs and OKCs presented similar ING4 labelling (Tables 2 and 3).

Table 2. Sample size, median, quartiles 25 and 75, mean of the ranks, Kruskal-Wallis statistic and statistical significance (p) for ING4 immunolabeling scores (cytoplasmic and nuclear labeling) in relation to the groups.

\begin{tabular}{|c|c|c|c|c|c|c|}
\hline Location/Groups & $\mathbf{N}$ & Median & $Q_{25}-Q_{75}$ & Mean of ranks & $\mathbf{H}$ & $\mathbf{P}$ \\
\hline \multicolumn{7}{|l|}{ Cytoplasm } \\
\hline $\mathrm{AM}$ & 20 & 2.00 & $1.00-3.00$ & 17.77 & 15.89 & $<0.001$ \\
\hline $\mathrm{OKC}$ & 20 & 1.00 & $2.00-3.00$ & 32.47 & & \\
\hline AOT & 15 & 2.00 & $2.00-3.00$ & 35.66 & & \\
\hline \multicolumn{7}{|l|}{ Nucleus } \\
\hline $\mathrm{AM}$ & 20 & 1.00 & $0.00-1.00$ & 17.5 & 20.61 & $<0.001$ \\
\hline $\mathrm{OKC}$ & 20 & 1.00 & $1.00-2.00$ & 28.6 & & \\
\hline AOT & 15 & 3.00 & $1.00-3.00$ & 41.2 & & \\
\hline
\end{tabular}

Legend: AM - ameloblastoma; OKC - odontogenic keratocyst; AOT - adenomatoid odontogenic tumor; H - Kruskal-Wallis coefficient.

Table 3. Sample size, median, quartiles 25 and 75, mean of the ranks, Mann-Whitney statistic and statistical significance (p) for ING4 immunolabeling scores (cytoplasmic and nuclear labeling) in relation to the groups when analyzed two-by-two.

\begin{tabular}{|c|c|c|c|c|c|c|}
\hline Location/Groups & $\mathbf{N}$ & Median & $\mathbf{Q}_{25}-\mathbf{Q}_{75}$ & Mean of ranks & $\mathbf{U}$ & $\mathbf{P}$ \\
\hline \multicolumn{7}{|l|}{ Cytoplasm } \\
\hline $\mathrm{AM}$ & 20 & 2.00 & $1.00-2.00$ & 15.075 & \multirow{2}{*}{91.5} & \multirow{2}{*}{0.001} \\
\hline $\mathrm{OKC}$ & 20 & 2.00 & $2.00-3.00$ & 25.925 & & \\
\hline $\mathrm{AM}$ & 20 & 2.00 & $1.00-2.00$ & 10.9 & \multirow{2}{*}{43.5} & \multirow{2}{*}{0.001} \\
\hline AOT & 15 & 2.00 & $2.00-3.00$ & 20.1 & & \\
\hline $\mathrm{OKC}$ & 20 & 2.00 & $2.00-3.00$ & 17.05 & \multirow{2}{*}{131} & \multirow{2}{*}{0.490} \\
\hline AOT & 15 & 2.00 & $2.00-3.00$ & 19.26 & & \\
\hline \multicolumn{7}{|l|}{ Nucleus } \\
\hline $\mathrm{AM}$ & 20 & 1.00 & $0.00-1.00$ & 16.05 & \multirow[t]{2}{*}{111} & \multirow[t]{2}{*}{0.01} \\
\hline $\mathrm{OKC}$ & 20 & 1.00 & $1.00-2.00$ & 24.95 & & \\
\hline AM & 20 & 1.00 & $0.00-1.00$ & 11.95 & \multirow[t]{2}{*}{29} & \multirow[t]{2}{*}{$<0.001$} \\
\hline AOT & 15 & 3.00 & $1.00-3.00$ & 26.06 & & \\
\hline $\mathrm{OKC}$ & 20 & 1.00 & $1.00-2.00$ & 14.15 & \multirow[t]{2}{*}{73} & \multirow[t]{2}{*}{0.007} \\
\hline AOT & 15 & 3.00 & $1.00-3.00$ & 23.13 & & \\
\hline
\end{tabular}

Legend: AM - ameloblastoma; OKC - odontogenic keratocyst; AOT - adenomatoid odontogenic tumor; U - Mann-Whitney Coefficient.

Considering nuclear immunolabeling analyses, AOTs showed higher ING4 scores compared to AMs ( $p$-value $<0.001)$ and OKCs $(p$-value $=0.007)$. OKCs presented higher values compared to AMs $(p$-value $=0.01)$ (Figure 3; Tables 2 and 3$)$. Thereby, statistically significant differences were observed, with AOTs exhibiting a higher median when compared to the $\mathrm{AM}$ and OKC groups. In addition, statistically significant differences were found between $\mathrm{AM}$ and $\mathrm{OKC}$, as well as between AM and AOT, and between OKC and AOT. 


\section{Discussion}

Odontogenic lesions present different degrees of aggressiveness and biological behavior. A wide range of epithelium associated factors have been implicated in the biological behavior of these lesions, including increased expression of various proliferative markers, impaired expression of tumor suppressor genes and their products, and abnormal cell cycle pathways. Studies on molecular events during odontogenesis have suggested that tumor suppressor genes are involved in odontogenic cyst and tumor development by modulating cell proliferation control (13). Hence, researchers have demonstrated that the state of ING-related mRNA expression is often altered in neoplastic tissue. This occurs for all members of the tumor suppressor ING gene family, including ING3 and ING4 $(7,14)$. In this context, the present study aimed to investigate the role of ING3 and ING4 proteins in the development of benign epithelial odontogenic lesions, as well as a possible relationship to biological behavior.

Although the World Health Organization classifies AMs and AOTs as epithelial odontogenic tumors, they show different biological behavior. Numerous factors have been associated with the aggressive behavior inherent to AMs, such as changes in the expression of tumor suppressor genes and their protein products (15). In previous studies, PCNA and p53 immunoexpression were evaluated in $\mathrm{AM}$ and AOT, and a significantly higher expression of p53 was detected in AMs, which could explain the more aggressive and invasive nature inherent to AMs (16, 17). In addition, p63 (a member of the p53 family) expression was evaluated in a group of epithelial odontogenic lesions. As a result, a comparable expression between OKCs and solid AMs was detected. Thus, the expression of p63 in cysts and tumors could be useful to assess the proliferative potential and aggressiveness of the lesions (18). Therefore, as observed in the present study, those findings reinforce that changes in the expression of tumor suppressor genes, such as p53 and ING family, are highly associated with the progression and biological behavior of odontogenic lesions.

In normal human tissues, ING3 expression has been positively correlated with growth rate, which suggests a possible role for ING3 in growth and self-renewal in certain diseases, such as cancer (19). Several mechanisms have been proposed to explain how the ING gene function is altered in tumors, which may include mutations arising from gene rearrangements, loss of heterozygosity (LOH), and loss of protein localization (20). The frequency of changes observed in ING3 and ING4 mRNA levels is due to genomic region allelic losses and reduced mRNA stability, among others factors. These factors allow irregular process combinations, which produce both reduced expression and function in these proteins (7). In the present study, ING3 immunolabeling was observed in the three lesions studied. Although no statistically significant differences were found between the groups, these findings are in line with studies demonstrating that increased cytoplasmic ING3 expression is related to nuclear expression losses in various neoplasms. This finding, resultant from nucleus-cytoplasmic translocation, would probably implicate on tumor progression $(7,9,21)$.

To fully perform its function, nuclear ING3 expression is required. Although the analysis revealed no statistically significant difference in ING3 nuclear labeling scores, our findings corroborate Borkosky et al. (22), which found LOH for this marker, which could probably be associated with a more aggressive AM phenotype. A previous study showed that LOH resulted in reduced ING3 nuclear expression in head and neck squamous cell carcinomas (23). Moreover, reduced ING3 nuclear immunolabeling is related to human melanoma progression and serves as a promising prognostic marker and therapeutic target for this malignancy (9). In the present study, some cases showed high cytoplasmic expression, in contrast to a low or absent nuclear expression. Therefore, in these situations, ING3 is probably unable to perform its functions. In this context, LOH could suppress ING3 functionality, leading to a higher susceptibility to the development of tumors, such as AM. In line with these findings, the present study also found loss of nuclear expression in AM, a clinically aggressive odontogenic tumor, and in OKC, which, when compared to other cysts and indolent tumors, such as AOT, also presents higher growth capacity. These results reinforce that reductions in ING3 expression may be associated with tumorigenesis and subsequent development of malignancies (7).

However, considering ING4 expression, we observed in the AOT group significantly higher frequencies of stronger immunolabeling, in contrast to the loss and reduction of immunolabeling in AM and OKC groups. ING4 overexpression disrupts cell cycle distribution, decreasing the number of cells in phase S. As a tumor suppressor gene, ING4 expression may suppress tumor growth in some neoplasms, such as glioma, breast cancer and head and neck squamous cells carcinoma (14). ING4 is known as a strong candidate involved in LOH in AMs, and its function is exerted primarily in cell nuclei, since the ING4 nuclear domain localization signal is required for interaction with p53 $(22,23)$. These 
observations pointed to control abnormalities in the AM and OKC group cell cycles. In addition, according to Borkosky et al. (22), the more aggressive and destructive behavior that characterizes ameloblastoma might be related to a more extensive inactivation in the tumor suppressor genes involved in this neoplasm, such as ING3 and ING4. This finding could explain the fact that we found a reduced or lost immunolabeling especially in $\mathrm{AM}$ and $\mathrm{OKC}$ groups.

On the other hand, considering the evidence of higher nuclear immunolabeling in less aggressive and indolent lesions, such as AOT, studies have suggested that ING4 may act in tumor suppression without affecting the cell cycle, yet regulating the transcription of genes involved in the control of multiple cellular functions (24). In normal conditions, ING4 mediates chromatin modification, has a suppressive effect on tumorigenesis, inhibits angiogenesis by suppressing the NF- $\mathrm{kB}$ pathway, and induces suppression of growth and apoptosis (25).

Therefore, the present study indicates that ING3 and, specially, ING4 downregulation may play an important role in the initiation and progression of more aggressive odontogenic cystic lesions, such as AMs and OKCs. Despite no statistical significance was verified for ING3 immunoexpression, we should not discard its probable influence on the development of these lesions. However, due to the scarcity of studies regarding the relationship between ING family proteins and odontogenic lesions, further studies are needed to better understand how these proteins influence on the biological behavior of odontogenic lesions.

\section{Acknowledgements}

The Brazilian National Council supported this study for Scientific and Technological Development (CNPq) (grant number 443341/2014-1).

\section{Resumo}

Objetivos: A família dos Genes Inibidores de Crescimento (ING) é um grupo de genes supressores tumorais que desempenham papéis importantes no controle do ciclo celular, na senescência, no reparo do DNA, na proliferação celular e na apoptose. No entanto, a inativação e a regulação negativa dessas proteínas têm sido relacionadas em algumas neoplasias. O objetivo do presente estudo foi avaliar o perfil imuno-histoquímico das proteínas ING3 e ING4 em uma série de lesões odontogênicas epiteliais benignas. Métodos: A amostra foi composta por espécimes de 20 ceratocistos odontogênicos (CO), 20 ameloblastomas (AM) e 15 tumores odontogênicos adenomatoides (TOA). A imunoexpressão nuclear e citoplasmática de ING3 e ING4 foram avaliadas semi-quantitativamente nas células epiteliais das lesões odontogênicas, de acordo com a porcentagem de células imunomarcadas em cada caso. As análises descritivas e estatísticas foram computadas, e o valor de $p$ estabelecido foi de 0,05 . Resultados: Não foram encontradas diferenças estatisticamente significativas na imunoexpressão citoplasmática e nuclear de ING3 entre as lesões estudadas. Em contrapartida, os TOAs apresentaram maior marcação citoplasmática e nuclear de ING4 em comparação aos AMs (valor de $p$ citoplasmático= 0,01 ; valor de $p$ nuclear $<0,001$ ) e COs (valor nuclear de $p=0,007$ ). Conclusão: A regulação negativa das proteínas ING3 e ING4 pode desempenhar um papel importante na iniciação e na progressão de lesões odontogênicas mais agressivas, como AMs e COs. 


\section{References}

1 - Avelar RL, Antunes AA, Carvalho RWF, Bezerra PGCF, Oliveira Neto PJ, Andrade ESS. Odontogenic cysts: a clinicopathological study of 507 cases. J Oral Sci 2009; 51(4):581-586.

2 - Mendenhall WM, Werning JW, Fernandes R, Malyapa RS, Mendenhall NP. Ameloblastoma. Am J Clin Oncol 2007; 30(6):645-648.

3 - Wu Y-H, Chang JY-F, Tseng C-H, Chiang C-P, Wang Y-P. Expression of astrocyte elevated gene-1 protein in ameloblastomas, keratocystic odontogenic tumors, and dentigerous cysts. J Oral Pathol Med 2017; 46(2):121-126.

4 - Premalatha BR, Patil S, Rao RS, Reddy NP, Indu M. Odontogenic tumor markers - an overview. J Int oral Heal 2013; 5(2):59-69.

5 - Guérillon C, Bigot N, Pedeux R. The ING tumor suppressor genes: Status in human tumors. Cancer Lett 2014; 345(1):1-16.

6 - Aguissa-Touré A-H, Wong RPC, Li G. The ING family tumor suppressors: from structure to function. Cell Mol Life Sci 2011; 68(1):45-54.

7 - Gou W-F, Yang X-F, Shen D-F, Zhao S, Sun H-Z, Luo J-S, et al. Immunohistochemical profile of ING3 protein in normal and cancerous tissues. Oncol Lett 2017; 13(3):1631-1636.

8 - Trinh DA, Shirakawa R, Kimura T, Sakata N, Goto K, Horiuchi H. Inhibitor of Growth 4 (ING4) is a positive regulator of rRNA synthesis. Sci Rep 2019; 9(1):1-14.

9 - Wang Y, Dai DL, Martinka M, Li G. Prognostic Significance of Nuclear ING3 Expression in Human Cutaneous Melanoma. Clin Cancer Res 2007; 13(14):4111-4116.

10 - Gunduz M, Nagatsuka H, Demircan K, Gunduz E, Cengiz B, Ouchida M, Tsujigiwa H, Yamachika E et al. Frequent deletion and down-regulation of ING4, a candidate tumor suppressor gene at 12p13, in head and neck squamous cell carcinomas. Gene 2005; 356:109-17.

11 - Tapia C, Zlobec I, Schneider S, Kilic E, Güth U, Bubendorf L, Kim S. Deletion of the inhibitor of growth 4 (ING4) tumor suppressor gene is prevalent in human epidermal growth factor 2 (HER2)positive breast cancer. Hum Pathol 2011; 42(7):983-90.

12 - Vered M, Peleg O, Taicher S, Buchner A. The immunoprofile of odontogenic keratocyst (keratocystic odontogenic tumor) that includes expression of PTCH, SMO, GLI-1 and bcl-2 is similar to ameloblastoma but different from odontogenic cysts. J Oral Pathol Med 2009; 38(7):597-604.

13 - Kwon H-JE, Jia S, Lan Y, Liu H, Jiang R. Activin and Bmp4 Signaling Converge on Wnt Activation during Odontogenesis. J Dent Res 2017; 96(10):1145-1152.

14 - Zhang L, Wang Y, Zhang F, Wang Y, Zhang Q. Correlation between tumor suppressor inhibitor of growth family member 4 expression and microvessel density in breast cancer. Hum Pathol 2012; 43(10):1611-1617.

15 - Kumamoto H, Izutsu T, Ohki K, Takahashi N, Ooya K. p53 gene status and expression of p53, MDM2, and p14ARF proteins in ameloblastomas. J Oral Pathol Med. 2004;33(5):292-299.

16 - Barboza CAG, Pinto LP, Freitas R de A, Costa A de LL, Souza LB de. Proliferating cell nuclear antigen (PCNA) and p53 protein expression in ameloblastoma and adenomatoid adontogenic tumor. Braz Dent J. 2005;16(1):56-61.

17 - Salehinejad J, Zare-Mahmoodabadi R, Saghafi S, et al. Immunohistochemical detection of p53 and PCNA in ameloblastoma and adenomatoid odontogenic tumor. J Oral Sci. 2011;53(2):213-217.

18 - Varsha B, Gharat Al, Nagamalini B, Jyothsna M, Mothkur S, Swaminathan U. Evaluation and comparison of expression of p63 in odontogenic keratocyst, solid ameloblastoma and unicystic ameloblastoma. J Oral Maxillofac Pathol. 2014;18(2):223.

19 - Nabbi A, Almami A, Thakur S, Suzuki K, Boland D, Bismar TA, Riabowol K. ING3 protein expression profiling in normal human tissues suggest its role in cellular growth and self-renewal. Eur $\mathrm{J}$ Cell Biol. 2015; 94(5):214-222.

20 - Gong W, Suzuki K, Russell M, Riabowol K. Function of the ING family of PHD proteins in cancer. Int J Biochem Cell Biol 2005; 37(5):1054-1065.

21 - Gunduz M, Ouchida M, Fukushima K, Ito S, Jitsumori Y, Nakashima T et al. Allelic loss and reduced expression of the ING3, a candidate tumor suppressor gene at 7q31, in human head and neck cancers. Oncogene 2002; 21(28):4462-4470.

22 - Borkosky SS, Gunduz M, Beder L, Tsujigiwa H, Tamamura R, Gunduz E, et al. Allelic Loss of the ING Gene Family Loci Is a Frequent Event in Ameloblastoma. Oncol Res Featur Preclin Clin Cancer Ther 2009; 18(10):509-18. 
23 - Raho G, Miranda C, Tamborini E, Pierotti MA, Greco A. Detection of novel mRNA splice variants of human ING4 tumor suppressor gene. Oncogene 2007; 26(36):5247-5257.

24 - Kim S. HuntING4 New Tumor Suppressors. Cell Cycle 2005; 4(4):516-7.

25 - Cai L, Li X, Zheng S, Wang Y, Wang Y, Li H, Yang J, Sun J. Inhibitor of growth 4 is involved in melanomagenesis and induces growth suppression and apoptosis in melanoma cell line M14. Melanoma Res 2009; 19(1):1-7.

Received: 05/02/2021

Accepted: 08/07/2021 